\title{
Г.А. Золотков
}

\section{«КОНЦЕПЦИЯ ЯЗЫКОВЫХ ИГР» КАК РЕШЕНИЕ ПРОБЛЕМЫ ПРИРОДЫ ФИЛОСОФИИ}

\begin{abstract}
Рассматривается представление об отсутствии у философии собственной предметной области, строящееся на концепции языка как совокупности автономных семантических систем. Прослеживаются негативные следствия этой позиции для таких составляюших природы философии, как её цель, метод и проблемы. Делается вывод о недостаточности принятия множественности семантических систем для решения проблемы природы философии.

Ключевые слова: концепщия языковых игр, проблема природы философии, метафилософский парадокс.
\end{abstract}

Прежде чем перейти к цели и задачам работы, несколько вводных замечаний. В настоящее время в философии языка широко обсуждается ряд таких позиций, как эпистемический контекстуализм, эпистемический плюрализм, эпистемический релятивизм ${ }^{1}$.

Эти позиции представлены разными группами исследователей и направлены на решение различных проблем. И всё же в них может быть усмотрено нечто родственное, а именно, лежащая в основе этих позиций концепция языка. Более того, можно предположить, что отличия между этими позициями происходят от выделения разных аспектов этой концепции. Она, следовательно, является одним из наиболее влиятельных взглядов на язык. Однако общепринятое название за ней пока не закрепилось.

Основная идея этой концепции заключается в постулате семантической дискретности языка. Говоря иначе, утверждается, что значения предложений устанавливаются не одним-единственным принципом, но множеством различных, а сам язык является не неким однородным целым, но совокупностью автономных языковых систем, в каждой из которых значения устанавливаются согласно нормам отдельной системы.

Привлекательность этой концепции языка объясняется способностью показать, почему, например, предложение «я не знаю» может иметь различные значения. Традиционно философия предпосылает языку целостность и один принцип установления значений, т.е. существование некоторого объективного значения, не зависящего от контекста. Недостатком такого шага является, например, то, что в рамках традиционных концепций предложение «я не знаю» имеет только одно отчётливое значение, а все остальные значения есть его искажения, значения которых всегда размыты. Очевидно, однако, что значения предложений повседневной речи, приводимые традиционалистами

\footnotetext{
${ }^{1}$ Среди участников этих дискуссий можно назвать Е. Лепура, К. Деросе, Дж. Дюпре, П. Богосяна (см. [3-6]).
} 
в качестве примера неточного использования языка, зачастую вполне однозначны. Например, предложение «я не знаю» как ответ на повседневный вопрос «мне идёт это платье?» имеет не менее чёткий смысл («не спрашивай меня»), чем если бы служило «объективному» указанию на отсутствие у говорящего знания, например, как в случае с вопросом «где моё платье?».

Принятие дискретности языка предлагает более удачное решение подобных проблем. Если допустить, что существует множество разных контекстов употребления «я не знаю», то каждое отдельное значение устанавливается уникальными правилами выбранной языковой системы. Дискретная концепция языка, разделяющая его на множество автономных систем, будет именоваться «концепцией языковых игр», сокращённо «КЯИ».

Название КЯИ отсылает к хрестоматийному примеру этой концепции, данному в поздних работах Л. Витгенштейна [1. С. 75-407]. Классический характер этих работ позволяет усмотреть КЯИ во многих актуальных тенденциях современной философии языка. С другой стороны, сделанная отсылка позволяет ввести уже устоявшийся терминологический аппарат: для обозначения автономной семантической системы - термин «языковая игра», для обозначения принципов наделения знаков этой системы значением - термин «правила».

Ниже будет рассмотрено приложение КЯИ к проблеме природы философии. Следует отметить, что даже в базовом варианте эта концепция предлагает мощное и в то же время простое решение этой проблемы. Пример её сокрушительной силы - рассмотрение философии Витгенштейна, ставящее под сомнение наличие у дисциплины собственной предметной области. Также не удивительно, что применение КЯИ к проблеме природы философии получило основное распространение в витгенштейнианстве [2] и в связанных с ним течениях. Автор, однако, предполагает, что для любого исследователя, принимающего КЯИ, естественным будет ход мысли, созвучный общему метафилософскому тону витгенштейнианства.

Цель работы - показать, что принятие КЯИ не необходимо влечёт отрицание предметной области, принадлежащей философии.

Рассмотрим метафилософский парадокс, который возникает при рассмотрении природы философии в КЯИ.

В общих чертах трактовка природы философии КЯИ состоит в следующем. Язык есть совокупность языковых игр, каждая из которых имеет собственные правила. Из этого представления о языке следует, что не существует универсальной системы правил, устанавливающей объективные значения. Традиционное стремление философии изложить универсальные правила есть ошибка, вызванная недопониманием природы языка. КЯИ склоняет философа ограничить свои умозрения реально существующими языковыми играми.

Если, однако, КЯИ верна, то в чём состоит отличие ви́дения правила языковой игры философом от видения этого же правила участником языковой игры? Так, последний осознаёт правило и действует согласно ему, философ осознаёт это правило таким же образом, но не следует ему, а анализирует его. Самого по 
себе этого описания не достаточно для разведения двух видений, так как очевидно, что на понимание правила не влияет то, следуют ему или нет.

Интуитивно видение правила философом представляется семантически более богатым. То есть оно должно дублировать стандартное видение, но добавлять к нему некоторые дополнительные значения. Поясним на примере. Представим, что некто осознаёт правило, но прокрастинирует, т.е. не торопится привести правило в исполнение. В этом случае видение правила не будет названо «философским», так как ему не хватает специфического осмысления правила. Если бы потребовалось переделать этот пример так, чтобы прокрастинатор стал философом, потребовалось бы добавить к его видению отсутствующие там значения.

Определение философии, стимулируемое принятием КЯИ и ставшее классическим, трактует философию как деятельность по анализу языка. Воспользуемся этим определением и предположим, что специфические значения синтезирует философский анализ языка. Не пытаясь дать определение этого анализа, сразу заметим, что возлагаемая на него функция синтеза новых для языковой игры значений противоречит КЯИ.

На первый взгляд, анализ имеет чёткую задачу - описать всё как есть, не добавляя ничего от себя, ограничиться извлечением значений из рассматриваемой им игры. Но в таком описании этот анализ, как было показано выше, теряет свою специфику и становится синонимичным прокрастинации.

Чтобы можно было сказать, что философский анализ раскрывает специфические значения, требуется указать на внешний источник этих значений. В противном случае останутся только значения, одинаково извлекаемые и тем, кто следует правилу, и тем, кто его анализирует. Также очевидно, что изложение правила как оно есть, без дополнительного осмысления, нельзя назвать философским анализом. Видение правила должно отличаться, так как ведёт к двум разным типам деятельности: следованию правилу и анализу правила.

Если же в самих языковых играх не содержится принцип их анализа, то он должен быть найден вне их. Запрет на осмысление языковых игр извне, устанавливаемый КЯИ, ведёт к невозможности философии синтезировать специфические значения.

Метафилософский парадокс КЯИ заключается в её неспособности объяснить, что служит источником специфики философского видения языковой игры. Языковая деятельность по созерцанию правил нуждается в дополнительных правилах, иначе теряется её отличие от обычного следования правилу.

Можно сформулировать этот парадокс в упрощённой форме. Проведём аналогию КЯИ с портретом семейства языковых игр. Такой портрет будет изображением всех форм осмысленной языковой деятельности, всё, что не попадает на портрет, - либо не языковая деятельность, либо лишено смысла. Будет ли на этом портрете изображена также и философия? По-видимому, нет. Философия это не языковая игра, у неё отсутствуют собственные правила. Будь они у неё, её анализ искажал бы смысл игр. Допустим, среди языковых игр её нет, но где-то она определённо должна быть. Парадокс КЯИ заключается в том, что никакого другого места для осмысленной языковой деятельности она не допускает. 
Рассмотрим подробнее, какие трудности указанный парадокс вызывает при решении проблемы природы философии. Для большей конкретности анализа примем стандартное деление этой проблемы на подпроблемы: цель, метод и природа проблем философии.

Традиционно целью философии считалось построение теорий, описывающих мир и мышление некоторым предельным образом. Если частные науки занимались развитием конкретных областей знания, то в философии обсуждалось знание вообще. Философия должна была синтезировать такое знание, истинность которого не зависела бы от контекста. Отрицание возможности такого знания выражается в принятии «негативной» и (или) «позитивной» цели философии.

«Негативная» цель философии состоит в устранении метафизических высказываний. Так, метафизики, объясняя значение таких концептов, как, например, концепт «знать», легко поддаются соблазну упростить себе задачу и дать обобщённое значение. Поступая так, метафизик отказывается от существующих употреблений этого концепта и вводит собственное. Такой ход позволяет ему освободиться от необходимости соблюдать конкретные языковые правила и объяснять концепт, используя те правила, которые он сам сочтёт нужным. «Негативная» цель философа, принимающего КЯИ, - произвести «терапию» метафизического употребления языка, т.е. показать, каким образом некоторое метафизическое высказывание нарушает правила.

Эта цель сталкивается с трудностью - чтобы осуществить терапию путаницы, её нужно сначала идентифицировать как путаницу. Сделать это можно, только указав, правила какой игры были нарушены. Например, метафизик использует для наделения значением некоторого понятия $p$ языковой игры $X$ не свойственные этому понятию правила языковой игры $Y$. Здесь вопрос вызывает то, идентифицирует философ, принявший КЯИ, путаницу потому, что были нарушены правила игры $X$ или правила игры $Y$ ? Каким бы ни был ответ, он предполагает принятие перспективы, рассматривающей сразу две игры, т.е. предлагает метапозицию. Отсюда следует, что принятие в КЯИ «негативной» цели опирается на возможность метапозиции.

«Позитивная» цель сводит философию к прояснению специфики отдельных игр. Если философ сам не должен изобретать новые правила, то он должен прояснять уже существующие.

Данная цель оправдывается тем, что выявить специфику языковой игры невозможно, пока её правила используются. Таким образом, чтобы следующий правилу мог осмыслить значение правила в рамках игры, ему нужно «поставить игру на паузу». Это позволит взглянуть на игру как на нечто целое, а не как на небольшое число актуально исполняемых правил. Тем не менее, чтобы представить игру целиком, требуется взглянуть на языковые игры извне. Кроме этого, чтобы можно было говорить о специфическом характере той или иной игры, требуется сравнить её с другими играми. Таким образом, данное определение цели философии, опять же, требует от философа занять метапозицию.

Традиционный метод философии в самом общем виде выглядит следующим образом: выдвигается метафизический тезис, затем следует его доказа- 
тельство. КЯИ определяет метафизические тезисы как бессмысленные и отрицает существование правил их доказательства. В качестве правильного метода философии КЯИ предлагает описание правил рассматриваемой игры, описание значений концептов или указание на неправильное их использование. Проще говоря, метод философии - это описание содержания языковых игр.

Основным преимуществом этого метода является обращение к реальным языковым правилам. Т.е. использующий этот метод всегда имеет перед глазами чёткий критерий легитимности своего анализа. Этим критерием являются действительные примеры работы языка, показывающие, верно ли описание или нет.

Основной трудностью, связанной с методом, понятым таким образом, является невозможность составить описание самого этого метода. Если КЯИ верна, то описано может быть только содержание языковых игр. Сам же этот метод не может иметь какого-то теоретически значимого содержания, но должен интуитивно схватываться через рассмотрение примеров его применения. То есть если в КЯИ делается утверждение, что существует метод философии, и этот метод - описание, то следует принять, что кроме языковых игр существуют нечто ещё, сообразно чему можно осуществлять философский анализ.

Традиционно природа проблем философии трактовалась как отражающая некоторое реальное положение дел. Например, онтологические проблемы описывают бытие, т.е. реально существующие феномены, например феномен «движение». КЯИ трактует природу подобных проблем как грамматическую, происходящую от неправильного употребления понятий. Смешение правил приводит к тому, что понятия типа «движение» начинают одновременно «отражать положение вещей» и «не отражать положение вещей». В КЯИ подобные высказывания лишаются какого-либо значения. Их природа целиком сводится к характерным языковым ошибкам, таким как излишнее обобщение, приписывание каждому существительному сущности, использование метафизического категориального аппарата. Резюмируя, философская проблема есть характерная языковая путаница.

Здесь следует подчеркнуть, что не каждая проблема определяется в КЯИ как философская. Допустим, некто задаётся вопросом, есть ли универсальные правила ношения на голове смешных колпаков. В другой раз этот же человек пытается понять, чем отличаются колпак и шляпа. В первом случае проблема имеет философский характер, во втором - нет. Отличить их позволяет, как уже было сказано, характерный тип совершаемой в первом случае ошибки.

Где, однако, можно найти перечень этих характерных ошибок? Его необходимо указать, чтобы понимание в рамках КЯИ концепта «философская проблема» было обоснованным. Кроме этого, сам этот перечень будет не чем иным, как специальным теоретическим аппаратом философии. Всё это ставит понимание природы философских проблем, вызываемое к жизни КЯИ, в зависимость от отрицаемых ею теоретических основ философии.

\section{4}

Выше были рассмотрены метафилософский парадокс КЯИ и те трудности, которые возникают при попытке решить проблему природы философии 
средствами этой концепции. Возможные следствия из этого парадокса и этих трудностей для проблемы природы философии могут быть следующими:

Первое возможное следствие. Философия является языковой игрой. Если исходить из того, что философия существует, а отрицание наличия у неё собственных правил ведёт к парадоксу и трудностям с определением целей, метода и природы философских проблем, то естественным шагом будет заключить об ошибочности отрицания специфических языковых правил философии.

Второе возможное следствие. Философия невозможна или бессмысленна. Этому, однако, противоречит как то, что КЯИ сама является философской концепцией, так и то, что тот факт, что философия существует, достаточно очевиден. С другой стороны, можно допустить, что философия существует как бессмысленная языковая деятельность. Но, опять же, это следует из самой философии, что приводит к парадоксу лжеца. Можно допустить, что устройство языка, на которое указывает КЯИ, истинно независимо от того, истинна ли сама КЯИ, но это не будет достаточным основанием для отрицания у философии содержания.

Третье возможное следствие. КЯИ не верна. Метафилософский парадокс и проблемы, возникающие при решении проблемы природы философии в рамках КЯИ, следует истолковать как доказательство неверности КЯИ.

Четвёртое возможное следствие. КЯИ не является абсолютной картиной языка, но должна быть дополнена, чтобы объяснить возможность философского взгляда на языковые игры. Таким образом, одновременно осуществляется принятие КЯИ и непринятие её решения проблемы природы философии. Действительно, интуитивно представляется верным, что язык является семейством языковых игр. В то же время следующая отсюда трактовка философа не как «специалиста в своём деле», но как «специалиста в чужих делах» представляется интуитивно неверной.

Таким образом, ни одно возможное следствие не допускает, что КЯИ позволяет решить проблему природы философии. Это позволяет сделать вывод о том, что принятие «КЯИ» не решает проблему природы философии, но, скорее, является способом вывести осмысление этой проблемы на новый уровень.

\section{Лumepamypa}

1. Витгенштейн Л. Философские работы. Часть І. М.: Гнозис, 1994. С. 75-321.

2. Золотков Г.А. Проблема природы философии в «ортодоксальном» витгенштейнианстве // Философский журнал. 2017. Т. 10, № 3. С. 61-74.

3. DeRose K. Solving the Skeptical Problem // The Philosophical Review. 1995. Vol. 104, No 1. P. $1-52$.

4. Cappelen H. and Lepore E. Insensitive Semantics. Oxford: Basil Blackwell Pub., 2005.

5. Boghossian P. Fear of Knowledge: against Relativism and Constructivism. Oxford: Clarendon Press, 2006.

6. Dupré J. The Disorder of Things. Metaphysical Foundations of the Disunity of Science. Cambridge, MA: Harvard University Press,. 1993.

Grigory A. Zolotkov. National Research University Higher School of Economics. (Moscow, Russian Federation)

E-mail: zolotkov_grigory@mail.ru

DOI: $10.17223 / 1998863 \mathrm{X} / 40 / 4$ 
'THE THEORY OF LANGUAGE-GAMES' AS THE SOLUTION OF THE PROBLEM OF THE NATURE OF PHILOSOPHY

Key words: theory of language-games, the problem of the nature of philosophy, the metaphilosophical paradox

The paper is concerned with metaphilosophical cases in which the assumption that there is no subject matter of philosophy is based on the position called 'the theory of language games'. In the contemporary philosophy of language there are a number of popular positions which based on the similar understanding of language. This popularity gives to the metaphilosophical paradox of that understanding and to the metaphilosophical difficulties which that understanding brings a certain value. The metaphilosophical paradox of 'the theory of language games' consists in the impossibility of that that understanding of language to explain the difference between the contemplation of the rule by a philosopher and the analogous contemplation by a rule-follower. That impossibility gives way to losing difference between two. The paradox could also be stated in the following way: the theory of language games' depicts the language in manner analogous to the way how a family portrait images a family. The paradox emerges from our attempts to place philosophy on that portrait. Also the paper provides the analysis of the difficulties which that understanding brings for the solving of the problem of philosophy. Using the example of the aim of philosophy, the method of philosophy and the nature of philosophical problems the author shows that 'the theory of language games' could say nothing noncontroversial about the nature of philosophy. The aim of philosophy according to 'the theory of language games' is or the removing of the philosophical confusions or the conceptual analysis. For both of them it is necessary to see language games from some meta-perspective. The right method of philosophy according to 'the theory of language games' is the describing of the language games. Such method to be pointed out should be based on some kind of rules. The nature of philosophical problems according to 'the theory of language games' is grammatical. Nevertheless, to describe something as the philosophical problem there should be given some theoretical basis. In the end author made a conclusion that 'the theory of language games' is not a sufficient position for solving the problem of the nature of philosophy.

\section{References}

1. Wittgenstein, L. (1994) Filosofskie raboty [Philosophical works]. Translated from German. Part I. Moscow: Gnozis. pp. 75-321.

2. Zolotkov, G.A. (2017) Problema prirody filosofii v "ortodoksal'nom" vitgenshteynianstve [The problem of the nature of philosophy in the "orthodox" Wittgensteinism]. Filosofskiy zhurnal. 10(3). pp. 61-74.

3. DeRose, K. (1995) Solving the Skeptical Problem. The Philosophical Review. 104(1). pp. 1-52.

4. Cappelen, H. \& Lepore, E. (2005) Insensitive Semantics. Oxford: Basil Blackwell Pub.

5. Boghossian, P. (2006) Fear of Knowledge: against Relativism and Constructivism. Oxford: Clarendon Press.

6. Dupré, J. (1993) The Disorder of Things. Metaphysical Foundations of the Disunity of Science. Cambridge, MA: Harvard University Press. 